\title{
Block copolymer structural color strain sensor
}

\author{
Tae Hyun Park', Seunggun Yu (1)', Sung Hwan Cho', Han Sol Kang', Yeongsik Kim², Min Ju Kim', Hongkyu Eoh', \\ Chanho Park', Beomjin Jeong ${ }^{1}$, Seung Won Lee', Du Yeol Ryu², June Huh ${ }^{3}$ and Cheolmin Park ${ }^{1}$
}

\begin{abstract}
The development of electrically responsive sensors based on the capacitance, voltage, and resistance that can detect and simultaneou sly visualize the large strain involved with human motion is in great demand. Here, we demonstrate a highly stretchable, large strain capacitive sensor that can visualize strain based on the strain-responsive structural color (SC). Our device contains an elastomeric sensing film that produces a capacitance change under strain, in which a selfassembled block copolymer (BCP) photonic crystal (PC) film with 1D periodic in-plane lamellae aligned parallel to the film surface is embedded for the efficient visualization of strain. The capacitance change arises from changes in the dimensions of the elastomer film under strain. The mechanochromic BCP PC film responds to strain, giving rise to an SC change with strain. The initial red SC of the sensor blueshifts and turns blue when the sensor is stretched to 100\%, resulting in a full-color SC alteration as a function of the strain. Our BCP SC strain sensor exhibits a fast strain response with multi-cycle reliability of both the capacitance and SC changes over 1000 cycles. This property allows for efficient visible recognition not only of the strained positions during finger bending and poking with a sharp object but also of the shapes of the strained objects.
\end{abstract}

\section{Introduction}

Strain and pressure sensors that are mechanically flexible, bendable, and stretchable are capable of being mounted on clothes and skin. These non-planar and deformable surfaces have attracted significant interest due to their potential for application in the detection of various physiological activities, health care monitoring, human motion detection, and diagnosis ${ }^{1,2}$. Numerous sensors have been demonstrated. The three major mechanical-to-electrical conversion principles are the capacitance $^{3-8}$, piezo-electricity ${ }^{9,10}$, and piezoresistivity ${ }^{11-17}$. Piezo-based sensors rely upon either electrical voltage or resistance changes under mechanical deformation. For instance, high-performance piezo-

Correspondence: Cheolmin Park (cmpark@yonsei.ac.kr)

${ }^{1}$ Department of Materials Science and Engineering, Yonsei University, Seoul, Republic of Korea

${ }^{2}$ Department of Chemical and Biomolecular Engineering, Yonsei University, Seoul, Republic of Korea

Full list of author information is available at the end of the article resistivity in 1D or 2D nanomaterials with efficient pressure-sensitive percolation of their networked structures upon pressure application has been employed. This property gave rise to a high pressure sensitivity ${ }^{12-14}$. On the other hand, capacitive sensors utilize capacitance changes in pressure-sensitive dielectric films under mechanical stimuli. These sensors are advantageous owing to their fast response times and compact circuit layouts with vertically stacked device architectures ${ }^{3-7}$. To make these sensors suitable for various applications, particularly for largely deformable strain detection, the sensing materials should be either elastomeric or efficiently embedded in a rubbery matrix. These requirements make the sensors tolerant to repetitive deformation ${ }^{6-8}$. Numerous previous works have been devoted to developing elastomer-based dielectrics for enhancing the sensitivity through efficient large-scale integration by combining a variety of structural architectures $^{3-5,8}$. These efforts have provided data supporting the relevance of these capacitive sensors. The sensor

\section{(c) The Author(s) 2018}

(c) Open Access This article is licensed under a Creative Commons Attribution 4.0 International License, which permits use, sharing, adaptation, distribution and reproduction cc) in any medium or format, as long as you give appropriate credit to the original author(s) and the source, provide a link to the Creative Commons license, and indicate if changes were made. The images or other third party material in this article are included in the article's Creative Commons license, unless indicated otherwise in a credit line to the material. If material is not included in the article's Creative Commons license and your intended use is not permitted by statutory regulation or exceeds the permitted use, you will need to obtain permission directly from the copyright holder. To view a copy of this license, visit http://creativecommons.org/licenses/by/4.0/. 
functionality was, however, limited to pressure and strain detection, thus precluding the realization of an interactive sensing capability that converts the stimulus to a humanreadable output.

The visualization of either a pressure or a strain source while sensing it can further extend the usefulness of a sensor by offering novel functions, such as shape and position recognition of a strained object and timedependent motion monitoring of an object on a sen$\operatorname{sor}^{3,5,6,9,10,14,17}$. This approach, commonly studied in the area of stimuli-interactive displays, utilizes the active matrix circuitry associated with active circuit elements such as transistors to provide precise spatial recognition ${ }^{3}$. For instance, tunable coloration/light emission have been successfully demonstrated by combining optical elements with pressure sensors, allowing for the visualization of pressure; examples include organic light emitting diodes $(\text { OLEDs })^{18}$ and electroluminescent ${ }^{19}$, electrochromic ${ }^{20}$, thermochromic ${ }^{21,22}$, and triboelectrification devices ${ }^{23}$. The visualization of a large strain of over $50 \%$ is required for wearable and skin-mountable sensors. However, this visualization is not straightforward and is possible with only a few mechanochromic systems that are sufficiently resistant to repetitive large strain deformation ${ }^{24,25}$. Despite the great success in visualizing large strain with mechanochromic films, no work has combined these films with electrically detectable strain sensors and human motion detection.

When a photonic crystal (PC) consisting of a periodic lattice arrangement of two different optical components is altered by strain, its structural colors (SCs) arising from the constructive interference of light with the periodic structure can be strain-dependent. This property gives rise to a useful method for the low-power reflective mode visualization of strain (Table S1, Supporting information $)^{24-35}$. In particular, SCs based on self-assembled block copolymers (BCPs) are more suitable, as their periodicities and dielectric constants are readily changed by mechanical forces such as shear, tensile, and compressive forces $^{24,25,29}$. For instance, a BCP film with a non-volatile plasticizing solvent as a domain swelling agent was successfully responsive to an approximately $100 \%$ strain imposed on the film ${ }^{24}$. We envisioned that a previously developed BCP soft solid with visible-range $\mathrm{SCs}^{36}$ could be suitable as the visualization component in a mechanically deformable large strain sensor when a thin, soft BCP SC film is appropriately embedded in an elastomeric medium. This approach is widely used to detect strain based on the strain-dependent capacitance change.

Here, we present a highly stretchable capacitive sensor with the ability to simultaneously visualize strain. For the visualization of the strain, a mechanochromic bilayer of ionic gel (IG) containing an ionic liquid as a BCP domain swelling agent and a BCP SC soft solid film with 1D periodic lamellae whose surface normal is perpendicular to the film surface is embedded in an elastomeric poly (dimethyl siloxane) (PDMS) polymer. Our device consisting of three vertically stacked components (hydrogel ionic conductor/BCP SC:IG in PDMS/hydrogel ionic conductor) successfully responds to strain through a strain-dependent capacitance change of the BCP SC:IG in the PDMS layer. At the same time, the BCP SC:IG film is strain-responsive. This property arises from an efficient change in the 1D lamellae structure under strain. The initial red SC of the sensor blueshifts and turns blue when fully stretched. This change gives rise to a full-color SC alteration as a function of strain. Our BCP SC strain sensor exhibits a fast strain response with a multi-cycle reliability in both the capacitance and the SC change over 1000 cycles, allowing for convenient visible recognition of finger bending, localized pressure and the shape of a strained object.

\section{Materials and methods Materials}

A symmetric polystyrene- $b$-poly(2-vinylpyridine) (PS- $b$ P2VP) block copolymer was synthesized via sequential living anionic polymerization in tetrahydrofuran (THF) at $-78^{\circ} \mathrm{C}$ using sec-butyllithium as an initiator ${ }^{37}$. The number-average molecular weight $\left(M_{n}\right)$ and polydispersity index $\left(\mathrm{PDI}=M_{w} / M_{n}\right)$, characterized by sizeexclusion chromatography (SEC), were measured to be $130 \mathrm{~kg} \mathrm{~mol}^{-1}$ and 1.03, respectively, as shown in Fig. S1. The PS volume fraction $\left(f_{P S}\right)$ of the BCP were determined to be 0.51 by ${ }^{1} \mathrm{H}$ nuclear magnetic resonance $\left({ }^{1} \mathrm{H}\right.$ NMR) spectroscopy (Fig. S2) based on the mass densities of the two blocks (1.05 and $1.14 \mathrm{~g} \mathrm{~cm}^{-3}$ for PS and P2VP, respectively). Propylene glycol monomethyl ether acetate, chloroform, bromoethane, hexane, 1,4-dibromobutane, and acetonitrile were purchased from Sigma-Aldrich. Poly (vinylidene fluoride-trifluoroethylene-chlorofluoroethylene) (PVDF-TrFE-CFE) containing $8 \mathrm{wt} \%$ CFE was purchased from Piezotech. Acrylamide (AAm; Sigma, A8887), N, N-methylenebisacrylamide (MBAA; Sigma, M7279), N,N,N ',N'-tetramethylethylenediamine (TEMED; Sigma, T7024), ammonium persulfate (AP; Sigma, A9164), and lithium chloride (LiCl; Sigma, L4408) were also purchased from Sigma-Aldrich and used to fabricate a hydrogel. VHB 4905 was purchased from $3 \mathrm{M}$ and used as received

\section{Preparation of BCP SC:IG Bilayers}

BCP SC films were prepared by spin-coating PS- $b$-P2VP block copolymer solutions (7 wt\% in propylene glycol monomethyl ether acetate) onto silicon wafers. The films were subsequently solvent-annealed in chloroform vapor at $60{ }^{\circ} \mathrm{C}$ for $12 \mathrm{~h}$. The P2VP domains of the BCP films were then selectively quaternized using 1-bromoethane and 1,4-dibromobutane in hexane at $60^{\circ} \mathrm{C}$ for $24 \mathrm{~h}$. The 

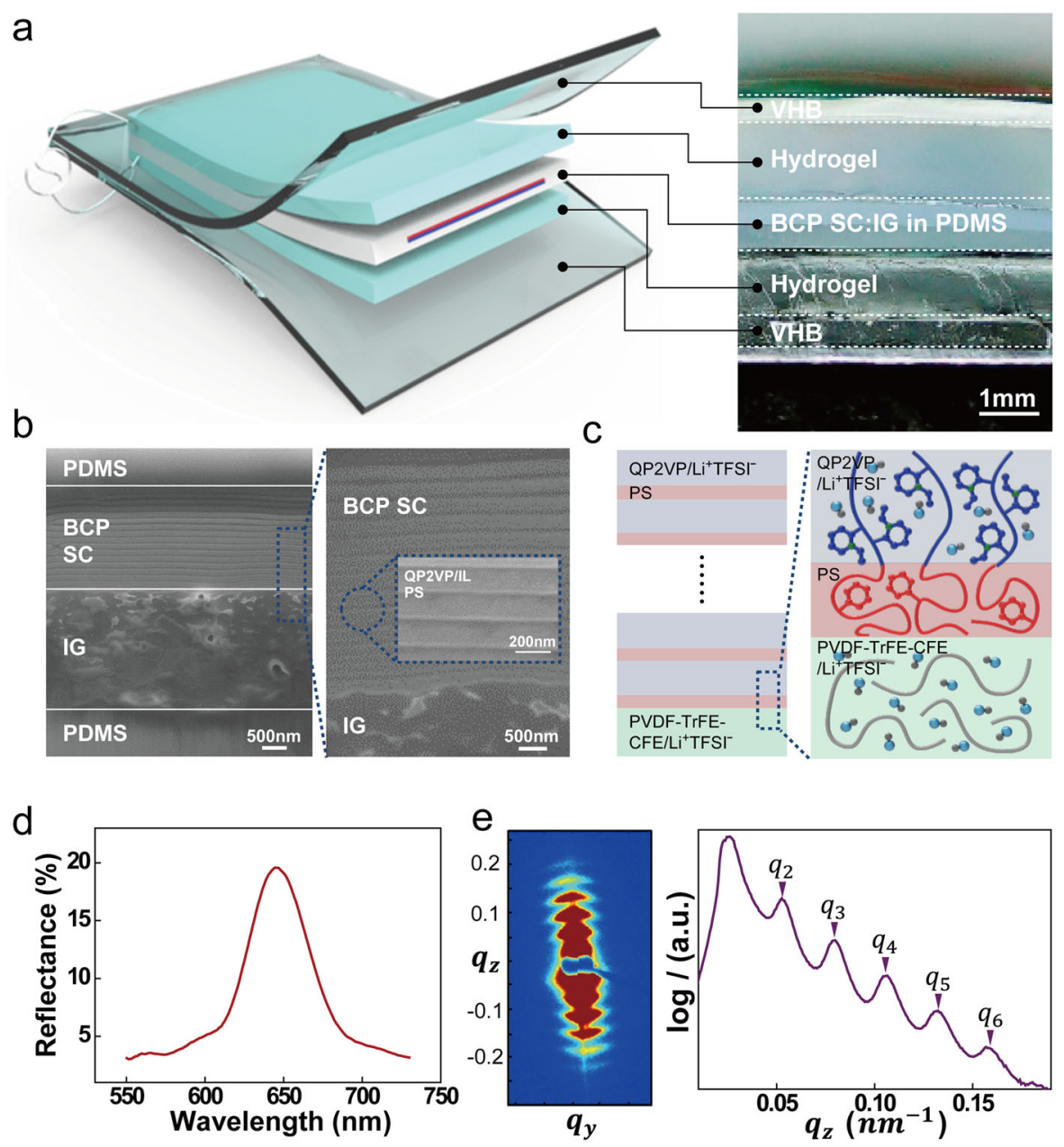

f

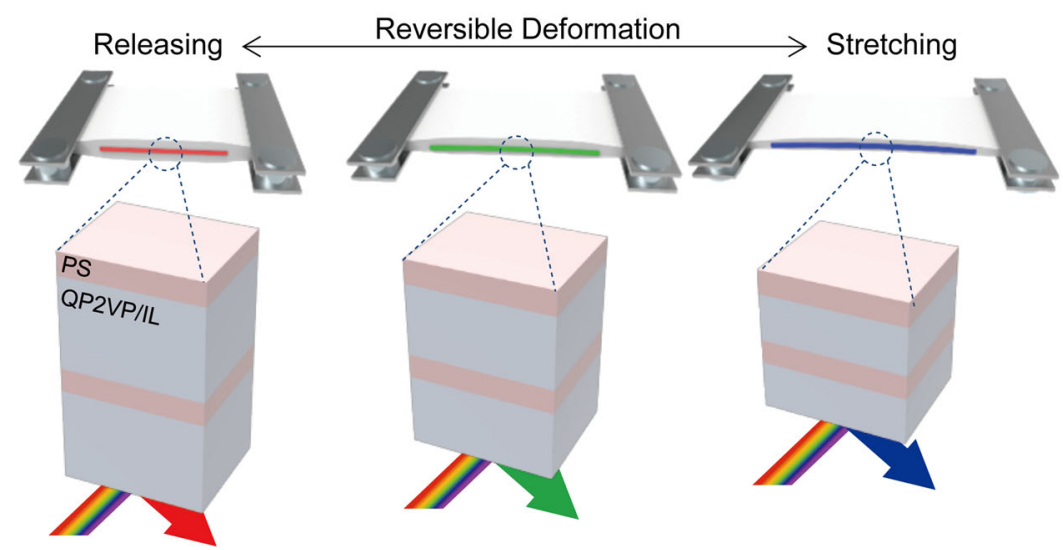

Fig. 1 a Schematic illustration of a BCP SC strain sensor. The sensor consists of VHB/hydrogel/BCP SC:IG in PDMS/hydrogel/VHB. Photograph of a cross-section of the sensor showing the layers vertically stacked on each other (right). $\mathbf{b}$ SEM image of a cross-sectioned BCP SC:IG in PDMS layer. The periodic BCP lamellae with the swollen QP2VP domains containing IL are visualized in the magnified images (right). c Schematic of the cross-section of a BCP SC:IG in PDMS layer. d UV-vis spectrum of a BCP SC sensor with its initial stop-band at a maximum wavelength of $650 \mathrm{~nm}$. e 2D SAXS pattern of BCP SC films embedded in PDMS with the incident beam perpendicular to the surface normal of the films. To achieve a sufficient scattering intensity, 10 BCP films were stacked on each other. On the right-hand side, a plot of the scattering intensity vs. the scattering vector $q_{z}$ is shown. $\mathbf{f}$ Schematic representing the variation in the SC of a BCP SC:IG film embedded in PDMS as a function of strain 
initial stopband of the BCP SC film was controlled by the degree of quaternization and the amount of 1,4-dibromobutane used during the quaternization process. IG layers consisting of PVDF-TrFE-CFE and $\mathrm{Li}^{+} \mathrm{TFSI}^{-}$were prepared by spin-coating onto a BCP film at 2000 r.p.m. for $60 \mathrm{~s}$.

\section{Preparation of BCP SC:IG Embedded in PDMS}

PDMS with a curing agent ratio of 20:1 was poured into half of a mold (width: $15 \mathrm{~mm}$, length: $70 \mathrm{~mm}$, thickness: 1 $\mathrm{mm}$ ) and pre-cured at $80^{\circ} \mathrm{C}$ after removing the bubbles with a vacuum pump. A BCP SC:IG layer was transferred to the pre-cured PDMS. Additional PDMS was subsequently poured onto the BCP SC:IG layer on the precured PDMS followed by thermal curing at $80^{\circ} \mathrm{C}$ for $3 \mathrm{~h}$.

\section{Fabrication of the BCP strain sensor}

The hydrogel was prepared as reported in the previous paper $^{7}$. The bottom ion-conductive hydrogel was cut into the desired shape and carefully placed on a VHB film. A BCP SC film embedded in PDMS was attached to the hydrogel. The top ion-conductive hydrogel was placed on the BCP SC:IG in PDMS, followed by attachment of another VHB layer to the top hydrogel to prevent the evaporation of water from the hydrogels.

\section{Characterization and Instrumentation}

The cross-sectional morphologies of the 1D in-plane lamellar structures in the BCP SC:IG in PDMS were imaged by TEM (JEM-F200) with thin cross-sectional samples prepared by a focused ion beam (FIB-4601F). 2D SAXS measurements were performed with 10 BCP SC films vertically stacked on each other to obtain a sufficient $\mathrm{X}$-ray scattering intensity using the PLS-II 9 A U-SAXS beamline at the Pohang Accelerator Laboratory. Reflectance spectra were taken by a UV-vis pectrometer (Ocean Optics USB2000, Filmetrics F40, and LAMBDA 750). A lab-made metal frame was used to stretch the BCP SC sensors during the reflectance measurements. The capacitance change measurements of the BCP SC strain sensors were performed with a precision LCR meter (Agilent E4980A) under ambient conditions. For the straindependent capacitance change measurements, a computer-controlled universal manipulator (Teraleader) was set up with the LCR meter.

\section{Results and discussion}

\section{Design and fabrication of a BCP SC strain sensor}

Our BCP SC strain sensor possesses a conventional capacitor structure with a mechanically responsive $\mathrm{BCP}$ SC:IG film in PDMS between two ion-conductive hydrogel electrodes physically protected with elastomeric VHB, as shown in Fig. 1a. The development of a BCP SC:IG film in PDMS started with fabricating a 1- $\mu \mathrm{m}-$ thick poly(styrene-block-2vinyl pyridine) (PS- $b$-P2VP) film with lamellae comprised of alternating PS and P2VP domains. A BCP solution in propylene glycol monomethyl ether acetate (PGMEA) was spin-coated. This step was followed by solvent annealing with chloroform vapor for $12 \mathrm{~h}$, which gave rise to a film with highly ordered inplane lamellae. Transmission electron microscopy (TEM) confirmed the formation of a stacked in-plane lamellae structure with a periodicity of approximately $60 \mathrm{~nm}$ (Fig. S3, Supporting information). Based on the nearly symmetric composition, both the PS and P2VP layers were $30 \mathrm{~nm}$ thick.

On the BCP film, which was quaternized and partially cross-linked through the pyridine rings of the P2VP blocks with a mixture of bromoethane and dibromobutane in hexane, a 3- $\mu$ m-thick IG layer was coated on a poly(vinylidene fluoride-co-trifluoroethylene-co-chlorofluoro ethylene) (PVDF-TrFE-CFE) film containing a quaternized P2VP domain swelling agent, $\mathrm{Li}^{+} \mathrm{TFSI}^{-36}$. The degree of cross-linking of the quaternized P2VP (QP2VP) domains was determined by the amount of dibromobutane. The characteristic SC of the BCP SC:IG film instantly appeared and was dependent upon not only the amount of $\mathrm{Li}^{+} \mathrm{TFSI}^{-}$in the IG layer but also the fraction of dibromobutane (Fig. S4, Supporting information). The BCP SC:IG film was subsequently embedded in an approximately $1000 \mu \mathrm{m}$ thick PDMS layer. A crosssection of the BCP SC:IG film embedded in PDMS prepared by a focused ion beam shows the periodic in-plane BCP lamellae with highly swollen QP2VP domains containing ionic liquid, as shown in Fig. 1b. The BCP SC:IG film embedded in PDMS is schematically illustrated in Fig. 1c. To electrically address the capacitance change of the PDMS containing the BCP SC:IG film under strain, two mechanically stretchable and ion-conductive hydrogel layers were placed on the top and bottom of the PDMS layer. Finally, the multilayered hydrogel/BCP SC:IG in PDMS/hydrogel structure was completely sealed in another rubbery matrix of VHB to prevent the evaporation of water from the hydrogel.

To utilize the full visible color range of the $\mathrm{SC}$ under strain, we initially tuned the SC of the BCP film to red with a wavelength at the maximum reflectivity of approximately $650 \mathrm{~nm}$, as shown in Fig. 1d. The average domain size of the swollen QP2VP of approximately 220 $\mathrm{nm}$ matches well to our finite-difference time-domain (FDTD) simulation in which the maximum reflection occurs at approximately $650 \mathrm{~nm}$ with periodic domains of QP2VP and PS of 220 and $30 \mathrm{~nm}$, respectively ${ }^{36}$. The inplane BCP lamellae were also confirmed by $2 \mathrm{D}$ smallangle X-ray scattering (SAXS) in transmittance mode, as shown in Fig. 1e. Spot-like multiple reflections appeared, which correspond to the 2nd-order, 3th-order, 4th-order, 5 th-order, and 6th-order reflections at scattering vectors 
of approximately $0.050,0.0769,0.1028,0.1273$, and 0.153 $\mathrm{nm}^{-1}$, respectively, at the meridian of the 2D SAXS pattern. The first-order refection was rarely detected because of parasitic scattering near the incident beam at very low $q$ regimes and was located at a scattering vector of approximately 0.025 , giving rise to expected values of the relative peak positions of the high-order reflections with respect to the first peak position $\left(q_{n} / q_{1}\right)$ of $1,2,3,4,5$, and 6 . The (100) plane distance obtained from $2 \pi / q_{1}$ was 251 $\mathrm{nm}$, which corresponded to the periodicity of the in-plane lamellae. This result was consistent with the microscope results shown in Fig. 1b. Note that to obtain sufficient scattering intensity, 10 layers of the $\mathrm{BCP}$ films swollen with ionic liquid were carefully stacked after removing the IG layers (Fig. S5, Supporting information). Our BCP SC strain sensor was then subjected to various mechanical deformations that could be quantitatively evaluated in terms of capacitance and SC changes, as schematically illustrated in Fig. 1f. The capacitance increased due to the decrease in the film thickness of the PDMS, and the stopband of the BCP SC blueshifted due to the decrease in the periodicity of the lamellae. One can anticipate that the capacitance change under strain may be dominated by dimensional changes in PDMS, which is 250 times thicker than the BCP SC:IG layer.

\section{Capacitive strain-sensing performance of a BCP SC sensor}

First, the capacitive sensing properties of our BCP SC sensor were examined, and the results are shown in Fig. 2. A capacitance change was observed in the sensor containing an approximately $4 \mu \mathrm{m}$ thick BCP SC:IG film embedded in a $1000 \mu \mathrm{m}$ thick PDMS with strain, as shown in Fig. 2a. The gauge factor $(G F)$ of the device was evaluated as a function of strain, which is defined as $G F=\delta$ $\left(\Delta C / C_{0}\right) / \delta \varepsilon$, where $\varepsilon$ is the applied strain, and $C$ and $C_{0}$ are the initial capacitances with and without applied strain, respectively. The results show that our sensor is capable of sensing a broad range of strain with a linear capacitance change as a function of the strain. The $G F$ value from the slope of the capacitance-strain plot was approximately 0.6, which is similar to those obtained in previous capacitive strain sensors based on dielectric rubbers such as PDMS ${ }^{6}$. Our BCP SC strain sensor promptly responds to consecutive multiple strains at $25 \%$ with fast response and relaxation times $(\sim 80 \mathrm{msec})$, as shown in Fig. $2 \mathrm{~b}$. The capacitance change response of our sensor to strain was sufficiently sensitive to detect knee and elbow motions in real time (Fig. S6, Supporting information). Figure 2c shows the repetitive capacitance cycling of the sensor under various applied strain ranges of 20,50 , and $100 \%$. We also evaluated the switching time constants as a function of the strain, and the results show that the time constants rarely change with strain (Fig. S7, Supporting information). Our device shows reliable capacitance response behavior during multiple strain/release cycles, in which the capacitance change was minimally altered, even after 5000 cycles with 50\% strain, as shown in Fig. 2d.

\section{Visualization of strain by BCP SC}

A BCP SC:IG film embedded in PDMS allows for facile visible color sensing based on its SC change, which arises from dimensional changes in the self-assembled lamellae under strain. This change gives rise to direct strain visualization simultaneously with capacitive sensing of the strain, as shown in Fig. 3. A sensor that exhibited an initial red SC with a maximum reflectivity at a wavelength of approximately $650 \mathrm{~nm}$ was stretched at a rate of approximately $0.05 \mathrm{~cm} / \mathrm{s}$. Our sensor was readily stretched up to approximately $100 \%$ and above, where it started to tear. The SC of the sensor clearly blueshifted with strain, as shown in the UV-vis reflectivity results provided in Fig. 3a. The applied strain exerted compression in the direction normal to the plane of the strain, which led to a decrease in the BCP SC:IG film thickness in PDMS. The wavelengths at the maximum reflectivity were plotted as a function of strain, and the results in Fig. 3b show that the values almost linearly decreased with the strain in the visible range. It should be noted that while the shift in the maximum position under strain mainly arises from the change in the average periodic distance of a BCP nanostructure under strain, the reflectance change is more complicated because it is affected by various factors, such as the local film thickness variation and local scattering. In fact, we employed an optical fiber-type UVvis spectrometer to characterize a small area of approximately $0.01 \mathrm{~mm}^{2}$ of the film. As described later, our BCP SC film contained numerous structural defects consisting of screw dislocations unevenly distributed in the film. The local scattering resulting from the screw dislocations was not uniform, and the film thickness also varied from point to point due to the unevenly distributed screw dislocations, giving rise to the variations in the reflectance.

The representative photographs of the sensor under the corresponding strain shown in Fig. 3c clearly suggest that the full visible range can be utilized for sensing a large strain of up to $100 \%$. It should also be noted that the local variations in the BCP SC observed in the photographs was mainly due to the presence of non-uniform BCP SC films. In fact, a completely flat and uniform BCP SC film was difficult to obtain during the transfer process in which a film prepared on a Si substrate was transferred to a PDMS layer, and instead, locally crumpled and irregular films were often achieved, giving rise to non-uniform SC, as shown in Fig. 3c. Another reason for the non-uniform SC is due to the screw dislocations that were unevenly distributed in the film, creating randomly located microscale cracks during repetitive strain events, as described in a later section. Our sensor was reliably stretched to $100 \%$ 

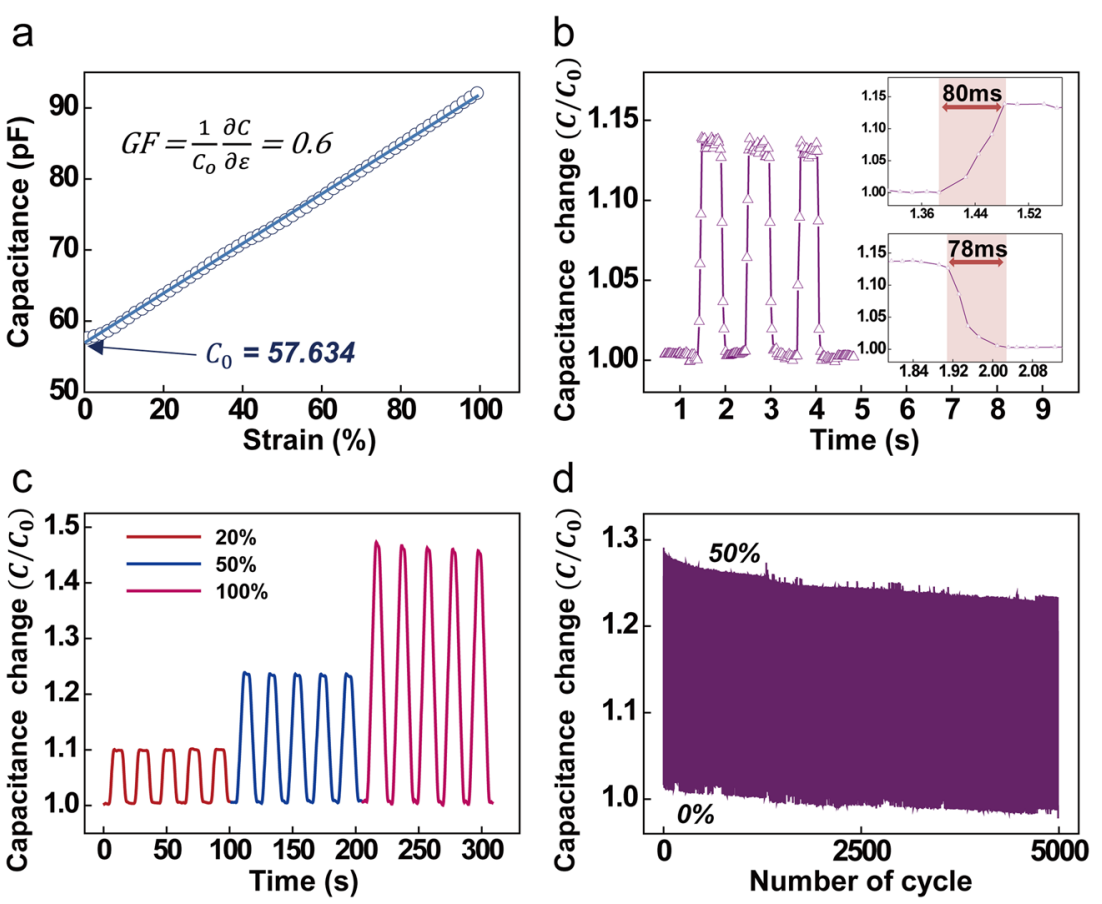

Fig. 2 a Plot of the capacitance vs. strain for a BCP SC sensor. b Capacitance change under 25\% strain with time. The insets show that the capacitance changes upon both stretching and release occur within $80 \mathrm{msec}$. c Capacitance changes during repetitive variations in the strain from 20 to $100 \%$. d Endurance of the capacitance change during more than 5000 50\% stretch/release cycles

and repeatedly recovered to its original shape. This cycle gave rise to repetitive switching of the SC from red to blue during multiple strain/release cycles, as shown in Fig. 3d. A reliable SC change during consecutive strain/release cycles was observed, as shown in Fig. 3e, where the initial $\mathrm{SC}$ with a wavelength at the maximum reflectivity of 650 $\mathrm{nm}$ barely changed during the 100 strain/release cycles. No significant degradation of the SC was observed, even after 1000 deformation cycles (Fig. S8, Supporting information).

To confirm that a decrease in the domain size occurred due to the external strain, SAXS experiments were performed, and the results are shown in Fig. 3f, g. 1D plots of a BCP SC in PDMS as a function of the strain obtained from the 2D SAXS results (Fig. S9, Supporting information) clearly show that the set of reflections shifted to higher $q$ values with strain. This indicates a decrease in the periodicity of the BCP film with strain. When the strain was released from the BCP film, the set of reflections recovered its original state without strain. It should be again noted that the first-order reflection peaks of the film under strain could not be detected because of parasitic scattering near the incident beam at very low $q$ regimes. The lamellar periodicity values from the firstorder reflections with different strains were 251,196 , and $174 \mathrm{~nm}$ for strains of 0,50 , and $100 \%$, respectively. The film after removing the strain exhibited as lamellar periodicity of 246 nm, as shown in Fig. 3g.

\section{Mechanism of the reversible visualization of strain in the BCP SC strain sensor}

The linear variation in the SC of the BCP SC:IG film with strain and the SAXS results suggest that affine deformation occurred in our sensor, and thus, the BCP lamellae with their initial periodicity decreased accordingly with the macroscopic strain. It is surprising that the periodic lamellae, particularly those having glassy PS domains that are minimally deformable with strain, underwent reversible large strain deformation. We revealed that the reversible deformation of the $\mathrm{BCP}$ films was attributed to two main factors: (1) the unique geometry of our BCP SC sensor, in which a BCP SC:IG film was embedded in an elastomeric PDMS and (2) the presence of an ionic liquid in the QP2VP domains. Note that all of the following experiments were carried out after removing the IG layer to clearly elucidate the deformation of the BCP film. A BCP film placed on a PDMS substrate without the ionic liquid was severely torn when stretched up to $100 \%$ strain in addition to the characteristic buckled structure with a periodicity of approximately 100 microns, as shown in Fig. 4a. When stretched by strain $\varepsilon_{s}$, a $\mathrm{BCP}$ film was compressed perpendicular to the strain direction $\left(F_{c}\right)$, resulting in a buckled structure along the strain 

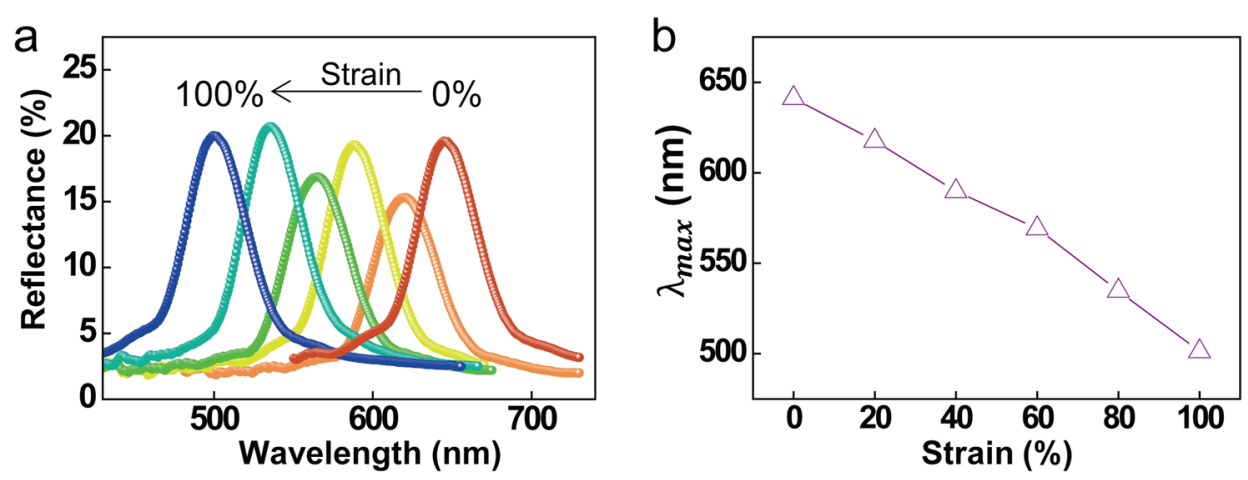

C
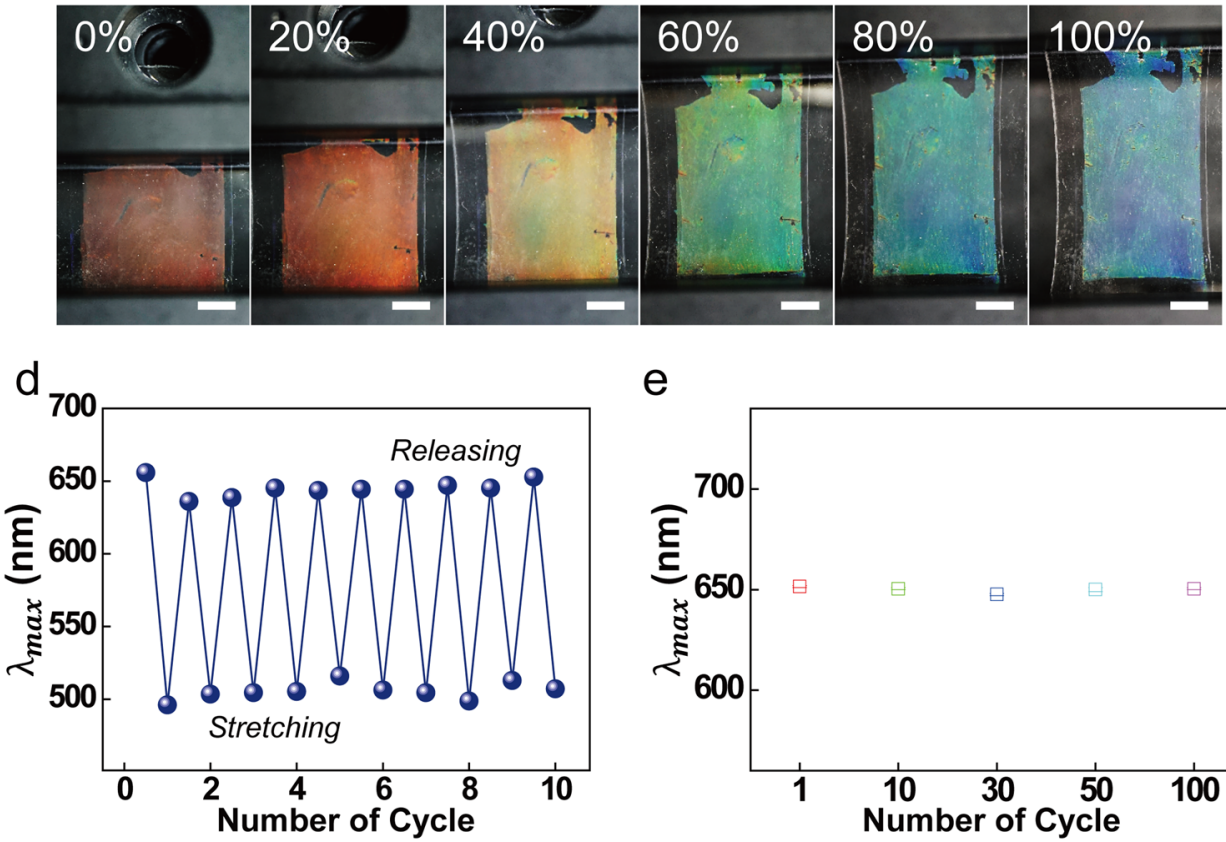

e

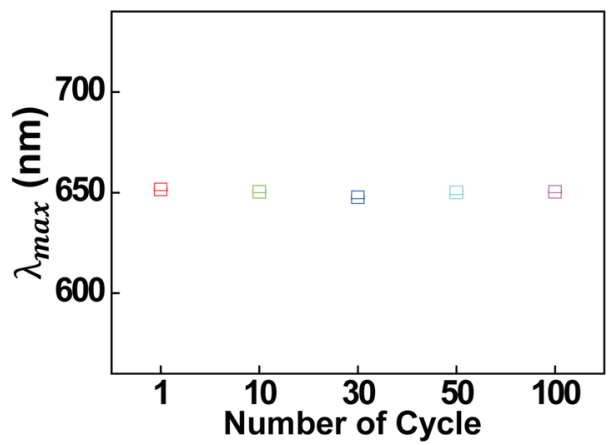

f

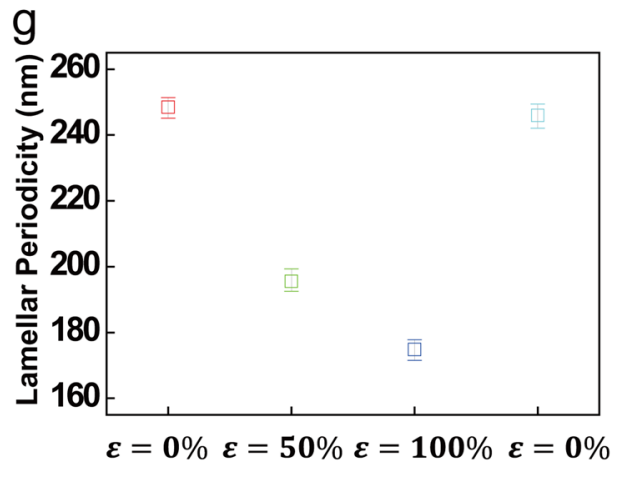

Fig. 3 a Reflectance spectra of a BCP SC sensor as a function of strain. The initial BCP SC without strain, which exhibits a wavelength at the maximum reflection of approximately $650 \mathrm{~nm}$, blueshifted with strain. b Plot of the wavelength at the maximum reflection as a function of strain. $\mathbf{c}$ Photographs of the sensor as a function of strain. All scale bars represent $1 \mathrm{~cm}$. $\mathbf{d}$ Wavelengths at the maximum reflection during repetitive stretching (100\% strain) and release cycles. e Variation in the wavelength at the maximum reflection with repetitive stretching (100\% strain) and release cycles. $\mathbf{f} 1 \mathrm{D}$ SAXS plots of the scattering intensity vs. the scattering vector $q_{z}$ of BCP SC films under different strains. To achieve a sufficient scattering intensity, 10 BCP films were stacked on each other. g Periodicities of the BCP lamellae under strains of 0,50, 100, and 0\% 


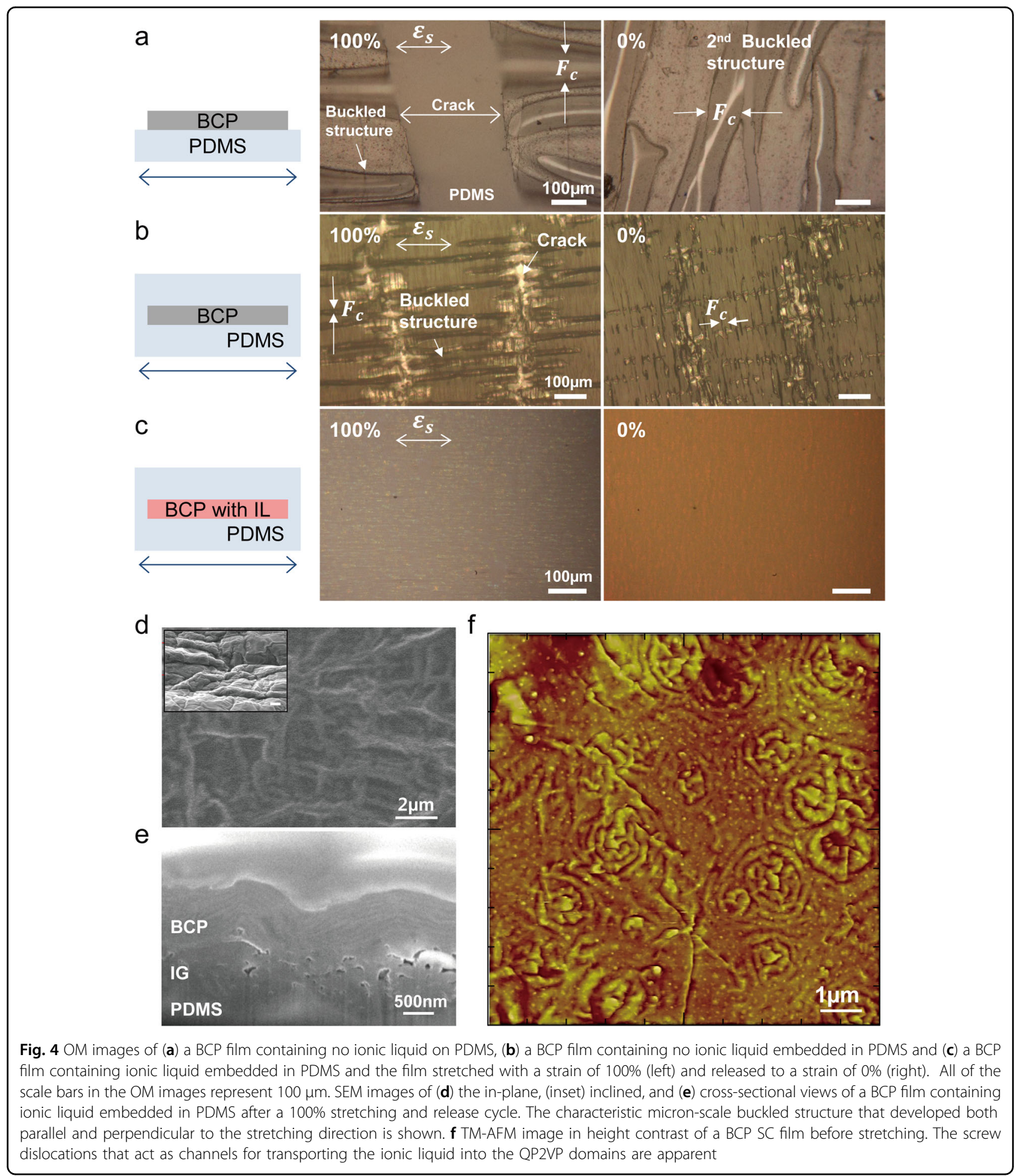

direction in some areas. When released to the 0\% strain state, another compressive force was exerted in the film parallel to the initial strain direction. A secondary buckled structure occurred in the film perpendicular to the compressive force. In contrast, mechanical failure was significantly reduced to give a buckled structure finer than that on PDMS when the BCP film was embedded in PDMS, as shown in Fig. 4b. This result implies that the confinement of the BCP film is beneficial for mitigating the mechanical failure of the film. The two types of buckled structures were also observed during stretch and release cycles. 
More importantly, the ionic liquid that is miscible with the QP2VP domains and causes them to swell plays a key role in reducing the mechanical failure during repetitive strain/release cycles when combined with the aforementioned unique confined geometry of the BCP film. Since the QP2VP domains become elastic due to the plasticization of the polymers with ionic liquid ${ }^{4,38}$, no large fracture of the film was observed, and the buckled structure was much finer when a BCP film with ionic liquid embedded in PDMS was stretched to $100 \%$ strain than that without ionic liquid, as shown in Fig. 4c. The secondary buckled structure also developed and was again much finer than that without ionic liquid, giving rise to two characteristic types of micro-buckled structures, as shown in Fig. 4d. A cross-sectional view of a deformed BCP film with ionic liquid also reveals a few-micron-scale buckled structure, as shown in Fig. 4e. A micro-buckled lamellae structure after the stretch/release cycle was also observed in the SAXS results, in which more broad reflections were observed after the cycle than for a film before deformation (Fig. S6, Supporting information). The film with the fine texture remained even after multiple strain/release cycles. This feature allowed for reliable and cyclic SC modulation.

What remains unclear is how the glassy PS domains periodically placed between the elastic QP2VP domains containing ionic liquid reliably respond to the $100 \%$ large strain/release cycles. The affine deformation of the PS domains that are immiscible with the ionic liquid is not plausible, and an alternative mechanical response to the strain should be proposed to explain the reversible SC modulation. In our BCP SC:IG film, the ionic liquid preferentially diffuses into the QP2VP domains proceeding from the top to the bottom of the micron-thick BCP film. The transport across the alternating PS and QP2VP in-plane layers occurs via the vertically oriented screw dislocations created while preparing the IG layer on the $\mathrm{BCP}$ layer. In our previous work, numerous screw dislocations a few micrometers in size developed in a BCP film ${ }^{39}$. When mechanical tensile stress is applied to a ductile film on an elastic substrate, the microcracks will deform slowly and withstand repeated loading and unloading cycles $^{40}$. We speculate that the centers of individual dislocations, which serve as channels for the ionic liquid, are responsible for efficient release of the mechanical stress exerted on the PS domains. Since mechanical rupture of the PS domains around the screw dislocations can occur rather evenly throughout the film under strain, it can be fully recovered when the strain is removed. We also identified the presence of screw dislocations a few micrometers in size uniformly distributed in the $\mathrm{BCP}$ film after detaching the IG layer from the $\mathrm{BCP}$ film, as shown in Fig. 4f. The micron-scale buckled structure may arise from the presence of screw dislocations in the film.

\section{Simultaneous sensing and visualization of external stimuli}

The great advantage of our BCP SC strain sensor lies in the fact that the strain exerted on the device can be directly visualized through the SC over a large strain. For instance, the large strain arising from a finger bending motion was readily detected via a capacitance change (Fig. 5a) with the clear visualization of the strain on the sensor, which changed from red to green with the bending strain, as shown in Fig. 5b. Another example of both sensing and visualization of large strain induced by pressure was demonstrated with our BCP SC sensor wrapped around a push-pull gauge with different forces, as shown in Fig. 5c. A blueshift in the sensor was observed due to the large strain. When our sensor that includes a $\mathrm{BCP}$ SC film is bent, the region of the film above the neutral plane is stretched, while that below the neutral plane is compressed. The SC usually blueshifts, which is mainly due to the stretching of the upper regions since compression rarely alters the $\mathrm{BCP}$ structure. The inconsistency in the SC around the circular lines with the largest strains in Fig. 5c arises mainly from the non-uniform strain applied to the BCP SC film (Fig. S10, Supporting information). At the same time, the capacitance properly responded to two forces of 4 and $8 \mathrm{~N}$, which again showed corresponding $\mathrm{SCs}$ of orange/green and blue, respectively.

Direct visualization of strain by our BCP SC sensor is also advantageous because it offers a convenient way to obtain positional information of strained areas without pixelated sensing arrays. Four different positions were pressed with a push-pull gauge having a contact area of approximately $0.0625 \pi \mathrm{mm}^{2}$ with the same pressure. While the capacitance change for all 4 pressing events was very similar due to the application of the same strain on the BCP SC sensor, the locations of the areas deformed by the cylinder were clearly visualized through the $\mathrm{SC}$, as shown in Fig. 5d. We also designed topographic clover, tree, star and stick rubber stamps with similar areas. When these stamps were gently touched to a BCP SC sensor with an area of $9 \mathrm{~cm}^{2}$, both capacitance and SC information were obtained. Figure $5 \mathrm{e}$ shows that the four different shapes were clearly visualized in the SC (left images in Fig. 5e), while no significant difference was observed in the capacitance since there was no pressure difference. The SC-based position and shape recognition is one of the most unique properties of our BCP SC sensor, which conventional strain sensors do not possess (Table S2, Supporting information). Our simple but robust BCP SC sensor based on a low-power reflective mode displays a promising capability to directly visualize the spatial distribution of strain on a single device. On the other hand, conventional strain visualization requires 

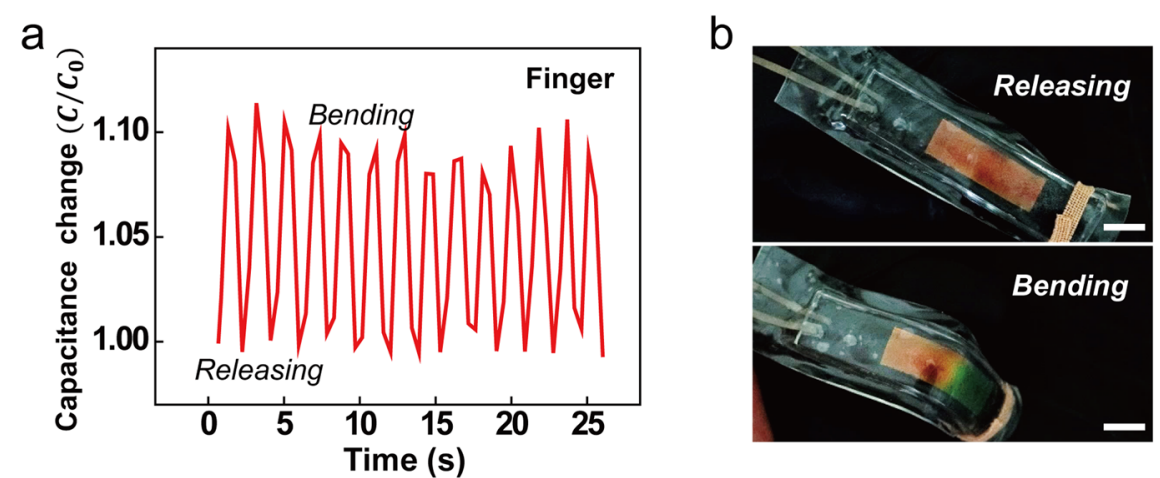

C

d

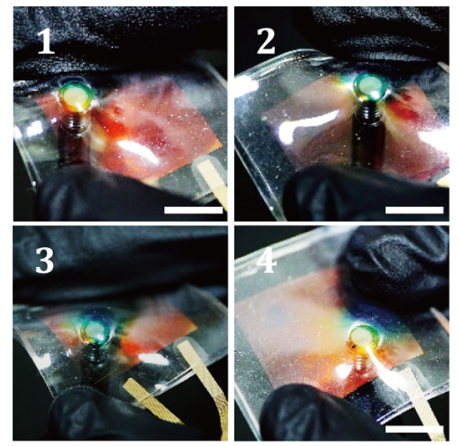

e

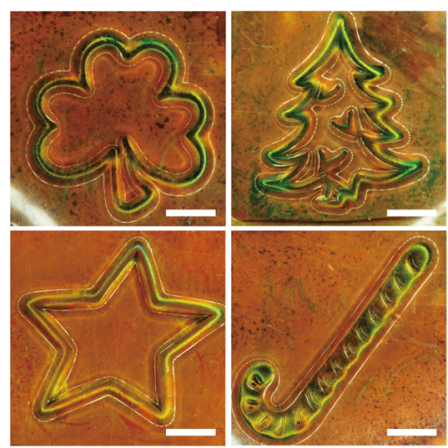

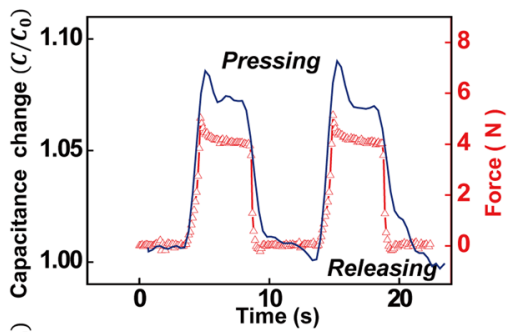
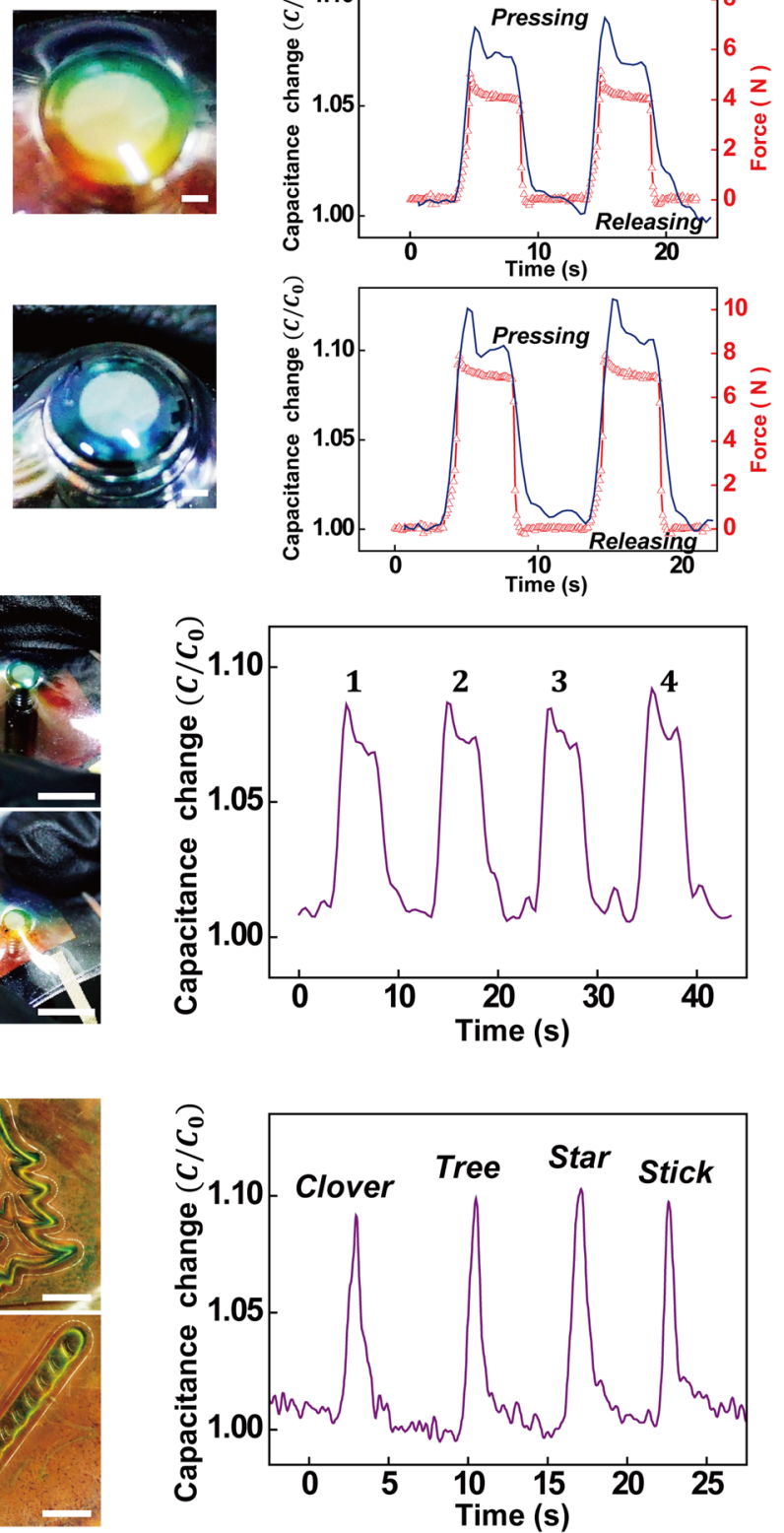
(see figure on previous page)

Fig. 5 a Capacitance changes upon multiple finger bending/release motions. b Photographs showing a BCP SC sensor mounted on a finger upon finger bending and release. $\mathbf{c}$ Photographs of a BCP SC sensor with an area of $3 \times 3 \mathrm{~cm}^{2}$ wrapped around a push-pull gauge with a pressure of 0,4 and, $8 \mathrm{~N}$. The capacitance changes upon wrapping with 4 and $8 \mathrm{~N}$ are shown in the plots on the right. $\mathbf{d}$ Photographs showing the device (c) strained by an $8 \mathrm{~N}$ pressure at different positions. The capacitance changes during the events corresponding to 1,2, 3, and 4 in the photographs on the left are shown on the right. e Photographs showing the devices pressurized with 4 different rubber stamps. The capacitance changes during the events on the left are shown on the right. All scale bars in (b), (c), (d) and (e) represent $1 \mathrm{~cm}$

high-density pixel arrays in display elements with complicated circuit integration to individually address spatial strain distributions.

\section{Conclusion}

We demonstrated that a novel BCP SC strain sensor is capable of electrically sensing strain through a capacitance change with simultaneous visualization of the strain. A thin mechanochromic bilayer of a BCP SC film with 1D periodic lamellae placed on an IG layer allowed for the direct visualization of strain while sensing the capacitance when embedded in an elastomeric PDMS dielectric medium. Our mechanically flexible and stretchable device consisting of a hydrogel ionic conductor/BCP SC:IG in PDMS/hydrogel ionic conductor structure exhibited a successful sensing performance, with both a capacitance change and an SC modulation observed as a function of strain up to $100 \%$. This result held true even after 1000 strain/release cycles. The affine compression of the 1D lamellae under strain caused the BCP SC to blueshift with strain, offering a useful route for visualizing the strain in full color. The characteristic micron-scale buckled structure of a BCP SC film with periodic lamellae containing glassy PS domains that developed during multiple cycles of deformation and recovery was responsible for the successful SC detection under large strain. This study revealed that the buckled structure arose not only from the unique confined geometry of the BCP SC: IG film in PDMS but also from the ionic liquid, which transported through the screw dislocations in the $\mathrm{BCP}$ film and caused the BCP domains to swell. Our BCP SC strain sensor was suitable as a reflectively visible electronic skin, which readily recognized human motion of the finger, elbow, and knee.

\section{Acknowledgements}

This research was supported by the Samsung Research Funding Center of Samsung Electronics under project number SRFC-MA1301-03.

\footnotetext{
Author details

'Department of Materials Science and Engineering, Yonsei University, Seoul, Republic of Korea. 'Department of Chemical and Biomolecular Engineering, Yonsei University, Seoul, Republic of Korea. ${ }^{3}$ Department of Chemical and Biological Engineering, Korea University, Seoul, Republic of Korea
}

\section{Conflict of interest}

The authors declare that they have no conflict of interest.

\section{Publisher's note}

Springer Nature remains neutral with regard to jurisdictional claims in published maps and institutional affiliations.

Supplementary information is available for this paper at https://doi.org/ 10.1038/s41427-018-0036-3.

Received: 20 September 2017 Revised: 14 November 2017 Accepted: 5 December 2017.

Published online: 18 April 2018

\section{References}

1. Amjadi, M., Kyung, K. U., Park, I. \& Sitti, M. Stretchable, skin-mountable, and wearable strain sensors and their potential applications: a review. Adv. Funct. Mater. 26, 1678-1698 (2016).

2. Zang, Y., Zhang, F., Di, C. \& Zhu, D. Advances of flexible pressure sensors toward artificial intelligence and health care applications. Mater. Horiz. 2, 140-156 (2015).

3. Mannsfeld, S. C. B. et al. Highly sensitive flexible pressure sensors with microstructured rubber dielectric layers. Nat. Mater. 9, 859-864 (2010).

4. Cho, S. H. et al. Micropatterned pyramidal ionic gels for sensing broad-range pressures with high sensitivity. ACS Appl. Mater. Interfaces 9, 10128-10135 (2017).

5. Nie, B., Li, R., Cao, J., Brandt, J. D. \& Pan, T. Flexible transparent iontronic film for interfacial capacitive pressure sensing. Adv. Mater. 27, 6055-6062 (2015).

6. Yao, S. \& Zhu, Y. Wearable multifunctional sensors using printed stretchable conductors made of silver nanowires. Nanoscale 6, 2345-2352 (2014).

7. Sun, J. Y., Keplinger, C., Whitesides, G. M. \& Suo, Z. Ionic skin. Adv. Mater. 26, 7608-7614 (2014)

8. Cohen, D. J., Mitra, D., Peterson, K. \& Maharbiz, M. M. A highly elastic, capacitive strain gauge based on percolating nanotube networks. Nano. Lett. 12, 1821-1825 (2012).

9. Wang, $X$. et al. Self-powered high-resolution and pressure-sensitive triboelectric sensor matrix for real-time tactile mapping. Adv. Mater. 28, 2896-2903 (2016).

10. Lin, L. et al. Triboelectric active sensor array for self-powered static and dynamic pressure detection and tactile imaging. ACS Nano 7, 8266-8274 (2013).

11. Kang, D. et al. Ultrasensitive mechanical crack-based sensor inspired by the spider sensory system. Nature 516, 222-226 (2014).

12. You, I. et al. Stretchable e-skin apexcardiogram sensor. Adv. Mater. 28, 6359-6364 (2016)

13. Chen, Z. et al. Enhancing the sensitivity of percolative graphene films for flexible and transparent pressure sensor arrays. Adv. Funct. Mater. 26 5061-5067 (2016).

14. Gong, S. et al. A wearable and highly sensitive pressure sensor with ultrathin gold nanowires. Nat. Commun. 5, 3132 (2014).

15. Amjadi, M., Pichitpajongkit, A., Lee, S., Ryu, S. \& Park, I. Highly stretchable and sensitive strain sensor based on silver-elastomer nanocomposite. ACS Nano $\mathbf{8}$, 5154-5163 (2014)

16. Yamada, T. et al. A stretchable carbon nanotube strain sensor for humanmotion detection. Nat. Nanotechnol. 6, 296-301 (2011). 
17. Pan, L. et al. An ultra-sensitive resistive pressure sensor based on hollowsphere microstructure induced elasticity in conducting polymer film. Nat. Commun. 5, 3002 (2014).

18. Wang, C. et al. User-interactive electronic skin for instantaneous pressure visualization. Nat. Mater. 12, 899 (2013).

19. Kim, E. H. et al. Organic light emitting board for dynamic interactive display. Nat. Commun. 8, 14964 (2017).

20. Chou, H.-H. et al. A chameleon-inspired stretchable electronic skin with interactive colour changing controlled by tactile sensing. Nat. Commun. $\mathbf{6}$, 8011 (2015).

21. Siegel, A. C., Phillips, S. T., Wiley, B. J. \& Whitesides, G. M. Thin, lightweight, foldable thermochromic displays on paper. Lab. Chip. 9, 2775-2781 (2009).

22. Kim, G. et al. Spatially pressure-mapped thermochromic interactive sensor. Adv. Mater. 29, 1606120 (2017).

23. Wang, $X$. et al. Full dynamic-range pressure sensor matrix based on optical and electrical dual-mode sensing. Adv. Mater. 29, 1605817 (2017).

24. Urbas, A. M. Block copolymer photonic crystals. Ph.D. Thesis, Massachusetts Institute of Technology, (2003).

25. Kolle, $M$. et al. Bio-inspired band-gap tunable elastic optical multilayer fibers. Adv. Mater. 25, 2239-2245 (2013).

26. Haque, M. A., Kamita, G., Kurokawa, T., Tsujii, K. \& Gong, J. P. Unidirectional alignment of lamellar bilayer in hydrogel: One-dimensional swelling, anisotropic modulus, and stress/strain tunable structural color. Adv. Mater. 22, 5110-5114 (2010).

27. Yue, $Y$. et al. Mechano-actuated ultrafast full-colour switching in layered photonic hydrogels. Nat. Commun. 5, 4659 (2014).

28. Howell, I. R., Li, C., Colella, N. S., Ito, K. \& Watkins, J. J. Strain-tunable one dimensional photonic crystals based on zirconium dioxide/slide-ring elastomer nanocomposites for mechanochromic sensing. ACS Appl. Mater. Interfaces 7, 3641-3646 (2015)
29. Chan, E. P., Walish, J. J., Thomas, E. L. \& Stafford, C. M. Block copolymer photonic gel for mechanochromic sensing. Adv. Mater. 23, 4702-4706 (2011)

30. Ge, D. et al. A robust smart window: Reversibly switching from high transparency to angle-independent structural color display. Adv. Mater. 27, 2489-2495 (2015)

31. Yang, D., Ye, S. \& Ge, J. From metastable colloidal crystalline arrays to fast responsive mechanochromic photonic gels: An organic gel for deformationbased display panels. Adv. Funct. Mater. 24, 3197-3205 (2014).

32. Fudouzi, H. \& Sawada, T. Photonic rubber sheets with tunable color by elastic deformation. Langmuir 22, 1365-1368 (2006).

33. Viel, B., Ruhl, T. \& Hellmann, G. P. Reversible deformation of opal elastomers Chem. Mater. 19, 5673-5679 (2007).

34. Arsenault, A. C. et al. From colour fingerprinting to the control of photoluminescence in elastic photonic crystals. Nat. Mater. 5, 179-184 (2006).

35. Chan, E. P., Walish, J. J., Urbas, A. M. \& Thomas, E. L. Mechanochromic photonic gels. Adv. Mater. 25, 3934-3947 (2013).

36. Park, T. J. et al. Electrically tunable soft-solid block. ACS Nano 9, 12158-12167 (2015).

37. Park, S. et al. Transition behavior of asymmetric polystyrene-b-poly(2-vinylpyridine) films: a stable hexagonally modulated layer structure. Polymer 60 32-39 (2015).

38. Scott, M. P. et al. Application of ionic liquids as plasticizers for poly(methyl methacrylate). Chem. Commun. 13, 1370-1371 (2002).

39. Kang, H. S. et al. Printable and rewritable full block copolymer structural color. Adv. Mater. 29, 1700084 (2017)

40. Lee, Y., Shin, M., Thiyagarajan, K. \& Jeong, U. Approaches to stretchable polymer active channels for deformable transistors. Macromolecules 49, 433-444 (2016). 